\title{
Disorder of bile acid synthesis
}

INSERM

\section{Source}

INSERM. (1999). Orphanet: an online rare disease and orphan drug data base. Disorder of bile acid synthesis. ORPHA:79168

Anomalies of bile acid synthesis are a group of sterol metabolism disorders due to enzyme deficiencies of bile acid synthesis (BAS) in infants, children and adults, with variable manifestations that include cholestasis, neurological disease, and fat malabsorption. Nine inborn errors have been described, 7 of which lead to liver cholestasis. 